\title{
Nuevas definiciones de evidencia en la Medicina contemporánea: aportes desde la Antropología
}

\section{New definitions of evidence in contemporary Medicine: contributions from Anthropology}

\author{
Juan Antonio Flores Martos \\ Universidad de Castilla-La Mancha. Facultad de Ciencias Sociales \\ de Talavera de la Reina. Departamento de Filosofía, Antropología, \\ Sociología y Estética. Toledo, España. \\ E-mail: juanantonio.floresळuclm.es \\ Lorenzo Mariano Juárez \\ Universidad Extremadura. Departamento de Enfermería. Cáceres, \\ España. \\ E-mail: lorenmarianoळunex.es
}

\section{Resumen}

Este artículo explora la importancia productiva de las Humanidades y de las Ciencias Sociales - en particular de la Antropología - a través de la revisión del "impacto" producido en un campo eminentemente empírico: la Medicina. El impacto de las Humanidades en general y la Antropología en particular tiene aquí una doble vertiente: 1) como base crítica epistemológica a una visión positivista "fundamentalista" - la enfermedad es algo meramente biológico - y 2) la incorporación de un nuevo repositorio humanístico en las prácticas contemporáneas. La creciente importancia de las Humanidades en la moderna medicina con la irrupción de la Medicina Basada en Narrativas, movimiento heredero de lo que se ha conocido como "Narrative Turn" en el campo de la Medicina - aquí ofrecemos un estudio de caso alrededor del Trastorno Límite de Personalidad - cuestiona las aseveraciones que insisten en su falta de productividad y eficacia. Proponemos aquí un movimiento de apertura hacia definiciones de evidencia más incluyentes - en la epistemología y la academia, pero también en la práctica clínica. Palabras clave: Antropología; Humanidades; Epistemología; Medicina Basada en Narrativas (MBN); Trastorno Límite de Personalidad (TLP).

\section{Correspondência}

Facultad de Enfermería y Terapia Ocupacional. Avenida de la U niversidad, s/n. Cáceres, 10003, España. 


\section{Abstract}

This article explores the "productive" importance of the Humanities and Social Sciences - Anthropology particularly - through the revision of the "impact" produced in an eminently empirical field: Medicine, particularly after the hegemony of Evidence Based Medicine (EBM). The impact of the Humanities in general and Anthropology in particular has a twofold meaning: 1) as a critical foundation against the "fundamentalistic" positivist view - disease is something purely biological - and 2) the incorporation of a new humanistic repository - maybe better the recovery of many of contemporary practices. The increasing relevance of Humanities on modern medicine with the emergence of Narrative-Based Medicine (NBM), movement of what has become known as "Narrative Turn" in the field of medicine - we offer here a case-study about Borderline Personality Disorder - challenges the assertions that insist on their lack of productivity and effectiveness. We suggest here an opening movement towards more inclusive definition of evidence-in epistemology and academia, but also in clinical practice.

Keywords: Anthropology; Humanities; Epistemology; Narrative-Based Medicine (NBM); Borderline Personality Disorder (BPD).
¿El ocaso contemporáneo de

\section{las Humanidades y las Ciencias} Sociales? Estertores en los tiempos de la evidencia y la patente

"El día que lo iban a matar..." De un tiempo a esta parte esta idea parece condensar el relato político e institucional sobre las Humanidades y las Ciencias Sociales (SSH). La crónica de una muerte anunciada. La noticia de la cancelación de los fondos de la National Science Foundation (NSF) ${ }^{2}$ para cualquier proyecto de investigación en el área de las Political-Science - a menos que demostrasen ser vitales para la seguridad nacional o los intereses económicos del país - sirvió para que Helga Nowotny publicara recientemente un artículo en The Guardian (2013) ${ }^{3}$ donde destacaba el prometedor futuro que aguardaba a las SSH en Europa, a diferencia del acoso que sufrían los colegas norteamericanos. Las afirmaciones de la presidenta del European Research Council fueron contestadas en una carta 4 firmada por diversos profesores de Universidades de toda Europa. Los autores destacaban las amenazas que se ciernen sobre la investigación en campos como la educación, el desempleo, la criminalidad, la migración, la herencia cultural o las artes dentro del programa de fondos (Framework Programme 7) en el Horizonte 2014-2020, con un severo recorte respecto al programa anterior, donde el porcentaje de fondos destinados a las SSH era casi marginal. Este escenario también se reproduce en los países de América del Sur - entre ellos Brasil -, cuyos contextos, pero sobre todo las políticas y lógicas (neoliberales, economicistas y medicalizadoras) de sus estados, exhiben similitudes importantes con los contextos europeos y español al que nos referiremos.

La crisis económica de los últimos años ha recrudecido una tendencia nada novedosa en las modernas sociedades postcapitalistas, donde las históricas tensiones entre las ciencias naturales

2 NSF cancels political-science grant cycle. Disponible en: <http://www.nature.com/news/nsf-cancels-political-science-grant-cycle-1.13501>. Acceso en: 9 ene. 2014 .

3 Shifting horizons for Europe's social sciences and humanities. Disponible en: <http://www.theguardian.com/science/political-science/2013/sep/23/europe-social-sciences-humanities>. Acceso en: 2 ago. 2013.

4 What future for research in social sciences and the humanities in Europe? Disponible en: <http://www.net4society.eu/_media/2013-10-29_ SSH_Article_What_Future.pdf $>$. Acceso en: 9 ene. 2014. 
y las "ciencias del espíritu" - en la distinción de Wilhelm Dilthey: "Naturwissenschaften" y "Geisteswissenschaft"-, han visto ensanchar las brechas y distancias entre las posiciones de hegemonía y subalternidad. Hasta hace no mucho el debate se articuló alrededor de asuntos de naturaleza epistemológica. Los defensores de la supremacía del empirismo desdeñaban los métodos de investigación y los resultados de los otros. Muestra, replicabilidad o fiabilidad se volvían asuntos sobre los que se dirimían las tensiones entre objetivistas y subjetivistas. Las Humanidades y las Ciencias Sociales se lanzaron a este campo de batalla con estrategias disímiles. Entre ellas, la construcción de una epistemología alternativa a la búsqueda empírica de Leyes pertrechada en la búsqueda de significaciones a través de Casos e Interpretaciones (Geertz, 1992). La distinción entre "Comprender" e "Interpretar" ofrecida por las Humanidades parecían suspirar por un espacio particular al modo del creado en los añejos debates teológicos entre Fe y Razón: a César y a Dios cada propiedad. La pérdida de los discursos legitimadores y el trabajo teórico a través de nociones como "reflexividad" invitaban a la ruptura de mundos pensados como hechos, desde entonces como interpretaciones, un fértil campo para la investigación de sociólogos, antropólogos o filósofos (Berger; Luckmann, 1986; Bourdieu, 1999). Décadas después, estas estrategias han sucumbido ante el pragmatismo contemporáneo, donde el impacto se ha medido en términos de eficacia económica y “empleabilidad". El espacio para las SSH es cada vez más angosto y parecen sonar campanas de duelo.

La estrategia frente al arrinconamiento ha sido en otras ocasiones la disputa dentro del campo del pragmatismo. Disciplinas como el Derecho encontraron capital en su campo de conocimiento en las cada vez más complejas sociedades postindustriales. Y hay quienes abrazaron la causa del empirismo, destacando el cientificismo de sus métodos y la eficacia y el valor de sus hallazgos, como la Sociología preocupada por las encuestas de opinión o la orientada al consumo. Incluso la Antropología, la más implícitamente revolucionaria de las ciencias humanas en palabras de Evens (1995), ofrece un fuerte compromiso con el valor aplicado de su conocimiento desde casi su fundación y las posibilidades para el control colonial (Malinowsky, 1929), y a lo largo de todo el siglo pasado en campos que han oscilado desde los conflictos bélicos, el espionaje o la cooperación al desarrollo.

Aparentemente, en vano. En el presente, como decíamos, los idus de marzo parecen haber llegado. Existe un vaciamiento del campo de las Humanidades - en las matrículas de alumnos de nuevo ingreso en sus facultades, las horas de sus asignaturas en la enseñanza secundaria- guiado desde las políticas educativas, los ministerios competentes y las directivas universitarias que enfatizan "la transferencia de la investigación" a la "sociedad" - entendiendo por ésta el tándem industria/empresa. Sólo existe el valor si hay alguien dispuesto a colocar un precio y pagarlo. A diferencia de lo que ocurre en otras tradiciones culturales, como la anglosajona o la francesa, el relato del valor del pensamiento crítico se percibe como accesorio o amenazante. Las Humanidades enfrentan un desafío que las conduce a una disyuntiva: reinvención (acaparar patentes) o disolución. La investigación, entendida del modo en que lo hacía Geertz, como un "pensativo paseo por una playa umbrosa" parece no tener hoy sitio (Geertz, 1989, p. 88).

Desde nuestra perspectiva, este planteamiento es erróneo, no sólo porque consideramos que el relato tradicional de las aportaciones de las Humanidades y las Ciencias Sociales continúa - quizás más que nunca - vigente. Más allá de esta evidencia, sostenemos que el impacto de las SSH tiende a ser menospreciado porque las escalas de evaluación - las patentes y los contratos con la empresa por ejemplo - no son las adecuadas. Las declaraciones de Peter Higgs ${ }^{5}$ después de haber recibido el premio nobel, donde comentaba apesadumbrado que en la actualidad él no sería suficientemente productivo para el sistema académico contemporáneo, son una muestra de la deriva de un sistema cuestionado. Para sostener esta tesis, este texto aborda el análisis del impacto de la producción de las SSH en el discurso y las prácticas

5 Peter Higgs: I wouldn't be productive enough for today's academic system. Disponible en: <http://www.theguardian.com/science/2013/ dec/o6/peter-higgs-boson-academic-system>. Acceso: 4 nov. 2013. 
en un campo de conocimiento anclado dentro del discurso de las ciencias empíricas: la Medicina. A través de una revisión de las aportaciones críticas de las SSH en las últimas décadas, pretendemos mostrar los giros - especialmente el giro narrativo - $y$ modificaciones de las epistemologías y las prácticas en éste dominio. Nos interesa identificar algunas expresiones alternativas del "impacto", el "valor" y el "pragmatismo" de las epistemologías hoy puestas en discusión.

La última sección del artículo, la dedicaremos a la exposición de un proyecto de investigación y primeros resultados - que llevamos a cabo con personas diagnosticadas con "Trastorno Límite de Personalidad" (Borderline), desde una perspectiva antropológica y priorizando el trabajo con narrativas. En ella ejemplificaremos los argumentos teóricos y metodológicos que constituyen el objeto de esta contribución. Ilustramos este giro narrativo epistemológico poniendo en primer plano los relatos de aflicción de estas personas - y sus familiares como una evidencia habitualmente ignorada por el paradigma hegemónico de la Medicina Basada en la Evidencia: la evidencia del sufrimiento. Ello supone trasladar el foco de la enfermedad de los neurotransmisores a la vida.

Desde un punto de vista epistemológico y metodológico, trabajar con estas narrativas e historias de vida nos permite elaborar un instrumento complementario y que es el reverso de los historiales clínicos convencionales. Además, entender estos relatos de aflicción como (nuevas) evidencias para la clínica nos ha permitido identificar la incomprensión que la experiencia de los pacientes refiere en las intervenciones profesionales, y disponer de un potencial para su conversión en documentos auditores de la calidad asistencial - y para la mejora de la atención, los cuidados y la eficacia.

\section{Medicina: una historia de ida y vuelta entre el Humanismo y la Física}

Aunque pocos lo recuerdan ya - además de historiadores y críticos de la medicina -, hasta hace no mucho tiempo lo que fue conocido como tekhné no era más que la labor artesanal del médico entendida como humanista. Desde finales del siglo XVIII, el devenir histórico de un proceso convenientemente descrito haría de la medicina una disciplina tan científica como la Física (Foucault, 2007; Rosen, 1985; Ackercknetch, 1986; Comelles, 2004). A pesar de los espectaculares avances tecnológicos y los éxitos cosechados por el modelo biomédico, una emergente propuesta - el giro narrativo y la Medicina Basada en Narrativas (MBN) - pretende redefinir la epistemología y la práctica médicas. Si la escuela anatomopatológica sentó las bases del denominado "giro copernicano en el pensamiento patológico" (Entralgo, 1978), donde lo invisible se hacía visible y la medicina un asunto de hechos y observables empíricos - la lesión dentro del cuerpo (Canguilhem, 1993, p. 76) -, asistimos en el presente a otro giro igual de sorprendente: los relatos de aflicción, la estadística cuando no es igual a uno, está empezando a ser considerada evidencia de primer orden. Y como aquí desarrollamos en la génesis de todo ello, se encuentran las aportaciones teóricas de las SSH; no se trata de un asunto insignificante.

El siglo XX, marcado por los avances tecnológicos y farmacológicos, desembocó en la ocupación de los espacios hegemónicos por parte de la biomedicina, imagen paradigmática de la retórica positivista y biologicista en lo que Menéndez denominó Modelo Médico Hegemónico. Hemos analizado la creciente valoración y la cada vez más notoria presencia de las humanidades y las ciencias sociales en las modernas definiciones de medicina. ¿Pero, cómo ha sido esto posible? ¿Qué ha sucedido para que la medicina, hasta hace bien poco el templo de la racionalidad científica y el espacio de los hechos irrefutables, gire de esta forma hacia las humanidades y la narrativa, entendidas ahora como evidencias de primer orden?

El modelo biomédico ha sido objeto de la crítica sociológica y antropológica, un proceso íntimamente relacionado con lo que en la literatura se ha denominado la crisis de la década de los sesenta y setenta (Illich, 1978; Menéndez, 1983, 1994). Las expectativas puestas en juego desde principios de siglo en el modelo curativo se derrumbaban sin que hubiera espacio para los modelos preventivos 
dentro de un discurso que negaba la subjetividad del paciente a costa de incrementar los costes y de la eficacia curativa, especialmente entre los pacientes crónicos y los terminales (Hunter, 1991). De aquel periodo surgieron las propuestas de reforma y acentuación de la Atención Primaria, el énfasis en una medicina holística de escucha al paciente (Bury, 2001, 1997) y los planes de prevención. Por otro lado, durante este tiempo el modelo alopático debía hacer frente al surgimiento de nuevos "estilos de vida" y el incremento de la expectativa de vida y los padecimientos crónicos-degenerativos, asuntos que trasladaron las demandas de salud del escenario de los procesos agudos y las cuestiones de vida o muerte al escenario crónico de la calidad de vida y la gestión del sufrimiento. La biomedicina era puesta en cuestión, y "no sólo la eficacia sino la ideología de la medicina denominada científica" (Menéndez, 1994, p. 79).

Sin que la medicina fuera aun consciente del todo, se abría paso el giro desde la necesidad de explicaciones y leyes a la necesidad de casos e interpretaciones. Y el contexto de emergencia de todo se hacía eco de los debates presentes en el seno de las Humanidades. En el contexto americano se asistía al descubrimiento de las culturas, la eclosión de las identidades y el multiculturalismo, proceso que acaecería en la década de los noventa en Europa. Una irrupción que amenazaba con socavar las visiones uniformes de unos cuerpos construidos en torno a la universalidad del biologicismo y su modelo de etiología única, que resultaría desde entonces un modelo explicativo insuficiente. Igualmente, la irrupción en el último cuarto del siglo pasado del paradigma posmoderno y la heteroglosia que le definía, va a tener un impacto claro en las nuevas epistemologías médicas, al menos con la crítica contemporánea. La mirada posmoderna se volvía esquiva a asumir un proceso investigador que "descubriera" verdades que esperaban agazapadas ser medidas y transformadas en reglas y regularidades, y asumía el carácter social de la construcción de la realidad y del conocimiento. De las metáforas propulsivas, el lenguaje de los pistones daba paso a las metáforas lúdicas y las analogías del drama (Geertz, 1994) para entrar de lleno en lo que la literatura ha denominado el "giro narrativo". El énfasis contemporáneo en las narrativas personales se acompañaba de un mayor conocimiento de los legos, una tendencia que no puede desligarse de la pérdida de autoridad de la ciencia y la medicina como "grandes narrativas" en el ordenamiento de la experiencia cotidiana y especialmente en las respuestas a los padecimientos. A finales del siglo pasado, la medicina había perdido el monopolio y el derecho exclusivo a hablar sobre el cuerpo y la enfermedad (Porter, 1997; Bury, 2001), inmersa en un proceso de democratización de recursos - medicinas alternativas, complementarias, recursos web, redes sociales etc. - que había reformulado el panorama médico. El posmoderno giro narrativo desplazaría el locus de la medicina del médico y su verdad factual en términos positivistas, a las historias de los pacientes y sus versiones y autoridades diversas.

Desde la década de los setenta, la enfermedad constituida como un locus apropiado para la investigación social ha proliferado de forma exponencial desde perspectivas simbólicas, interpretativas o hermenéuticas. Los trabajos pioneros de los antropólogos de Harvard Kleinman (1973, 1980) y Good (1977) transformaron los tradicionales enfoques biologicistas sobre la enfermedad, reconvertida desde entonces en sentido a través de conceptos cada vez más usuales, como símbolo, metáfora, narrativa, "mundo moral local" o "red semántica de enfermedad”. La línea de estos trabajos se alejaría entonces de los enfoques centrados en "explicaciones" (Erklarung) hacia modos de conocimientos ligados a la comprensión (Verstehen) y la interpretación (Auslegung). Del "ideal de explicaciones de leyes-y-ejemplos hacia otro ideal de casos-e-interpretaciones" (Geertz, 1991, p. 63). La enfermedad abandonaba el espacio de la naturaleza para llenarse de cultura (Perdiguero; Comelles, 200o) definida entonces como símbolos o textos interpretables en términos de contexto (Hernáez, 20o8, p. 83).

El impacto en la práctica médica de todas estas críticas fue entonces inapreciable. La medicina respondería con su particular "prietas las filas” en la década de los ochenta, cuando la culminación del movimiento empirista de la medicina se alcanzaría con la irrupción del paradigma de la Medicina Ba- 
sada en la Evidencia - MBE (Guyatt, 2002; Sackett, 1992, 2009; Sackett et al., 1996, 1997). Bajo el clásico lema de "facts, just give us the facts", la medicina consolidaba el giro cientificista, reduciendo el asunto de los problemas de salud y enfermedad a la esfera de los ensayos clínicos, los protocolos y la investigación terapéutica. Todo lo que excediera estos ámbitos sería revestido con el aura de la sospecha. La medicina cumplía el sueño edípico de matar al padre: las humanidades no tenían cabida (al menos hasta mediados de la década de los noventa).

\section{La moderna herencia humanística: narrative-based medicine}

En estos años ${ }^{6}$ vieron la luz de forma sistemática una serie de publicaciones empeñadas en rescatar el valor de todo lo que había sido excluido en aquella revitalización del paradigma positivista (Evans; Sweeney, 1998). La Medicina Basada en Narrativas emergía como una corriente que pretendía rescatar la narrativa y experiencia del paciente, intentando emprender la función de "puente" entre médicos y pacientes, acortando la distancia entre "saber" acerca de la enfermedad y "comprender" la experiencia del paciente (Greenhalgh; Hurwitz, 1998; Charon, 1993b, 2001, 2006; Juárez, 2011) al definir y asumir ciertos límites de la objetividad en el método clínico y el ejercicio diagnóstico, un asunto menos de "evidencias” en términos de ensayos clínicos y más de historias interpretadas (Greenhalgh, 1999; Juárez; Martos; Cipriano, 2013). Este es el giro narrativo de la medicina.

Este marco teórico partía de una desmitificación del mundo de hechos y observables empíricos de la clínica para ofrecer espacio al mundo subjetivo de la experiencia del paciente, entendiendo la narrativa como "aparatos semiológicos que producen significados" y abriendo el espectro de "evidencias" a asuntos como dolor interior, la esperanza y la desesperación, el dolor moral "que frecuentemente acompañan y a menudo, de hecho constituyen las enfermedades que sufren las personas" (Greenhalgh; Hurwitz, 1998). Los relatos de la experiencia de los pacientes pasaban a considerarse como "evidencias" y su producción bibliográfica crecía dentro de los estudios médicos (Aronson, 200o).

Tal y como han señalado Juárez, Rodríguez y Conde (2013), la revalorización de la palabra se produjo a través de "modelos teóricos que ensalzaban el acto de lectura y escritura de las narrativas de los pacientes, que debían ser escuchados de una manera "activa” (Kalitzkus, 2009), abarcando “oceánicamente" toda la narrativa, en la línea de lo que Kleinman (1988, p. 54) desde la Antropología había denominado una postura de "testigo empático" (empathetic witnessing).

Los cambios se producían también en la posición del galeno frente al paciente. Si desde la década de los sesenta se había definido un ideal de práctica médica basada en el distanciamiento - "detached concern" -, el renovado paradigma afirma la necesidad de una práctica esencialmente comprometida - "engaged concern" - (Halpern, 2001; Charon, 1993b, 2003; Hurwitz, 200o). Practicar la medicina no es un simple ejercicio de "saber acerca" y "descubrir" lo que sucede expresado en forma de diagnóstico, sino vivir desde dentro, acompañar al paciente en su padecimiento - etimológicamente, en su pathos con la enfermedad, esto es, "Living Through".

Por otra parte, ese alegato a los mundos emocionales, al carácter cualitativo de la práctica clínica y una atención a variables ciertamente poco o nada "mensurables" implicaba también una puesta en valor de lo concreto, una defensa a la importancia de una epidemiología cuando $n=1$. Los profesionales deberían huir de los valores promedio, en palabras de Bruner (1986), "subjuntivando" la realidad, pasando del modo indicativo al subjuntivo, esto es, al igual que la literatura, no reflejar la realidad sino ofrecer

6 Con una génesis contestataria a la MBE (EBM) las relaboraciones de las propuestas "clásicas" de la MBN (NBM) se canalizan en la actualidad en diversos grupos de trabajo (Silva et al., 2010; Charon et al., 2008) que persiguen construir un modelo clínico que dé cabida tanto a las aproximaciones positivistas como a las competencias narrativas con implicaciones para el desarrollo de la práctica terapéutica y la definición de los cuidados, los planes de ordenación y los currículums docentes y los nuevos marcos de investigación. Definida entonces como "Narrative Evidence Based Medicine" (NBM), el objetivo es construir un puente entre los estudios clínicos y el arte médico de aplicar el conocimiento a un caso individual, de tal manera que la EBM y la NBM deberían ser entendidas en términos complementarios (Kalitzkus, 2009, p. 81). 
sentido, "dotar de extrañeza a lo familiar, ir de lo que es a lo que podría" (Carrió, 2008), disminuyendo de esta forma los posibles errores. Haciendo suyas muchas de las críticas vertidas sobre la ortodoxia de la EBN, defendieron que los estudios de cohorte no podían ser trasladados sin reflexión a los casos particulares, un proceso imposible sin tener en cuenta el contexto. El giro narrativo conduciría a la definición de la narrativa - y la palabra - como evidencia más allá de los ensayos clínicos (Kalitzkus, 2009, p. 80). La NBM se mueve así de forma compleja entre las tensiones de la generalización y los grandes números y la necesidad de concreción en biografías particulares.

El peso de la crítica de las ciencias sociales y las humanidades en todo este movimiento es evidente. El convulso momento en la actual epistemología médica - incipiente aún en la práctica - bebe de diversas aproximaciones constructivistas e interpretativas a los procesos de salud y enfermedad desde la década de los setenta. Como modelo teórico que busca un reflejo en la práctica, la medicina basada en narrativas se vuelca en las humanidades, especialmente en la literatura (Charon, 1993a), a través de seminarios donde se pide a los alumnos escribir en un lenguaje no técnico destapando sus propios sentimientos, grupos de lectura sobre relatos illness o clásicos como Cortázar, Faulkner o Henry James y donde la bibliografía sobre los actos narrativos son un lugar común de esta formación en hospitales, definiendo las bases de la moderna "competencia narrativa".

Las pretensiones de una Medicina centrada en el paciente reposan en los marcos teóricos otorgados en las SSH al concepto de experiencia, que si bien había sido relevante con anterioridad, alcanzaría una posición protagonista en la década de los ochenta desde enfoques que privilegiaron el análisis de las prácticas sociales y las vivencias cotidianas de los individuos o los procesos de articulación identitarios de la experiencia dentro de los contextos socioculturales (Turner; Bruner, 1986; Comaroff; Comaroff, 1987). Los trabajos articulados en torno a la "fenomenología de la experiencia de la enfermedad" se han erigido como importantes medios para el análisis de la relación entre significado y experiencia como fenómenos interpretativos. Desde esta perspectiva, de acuerdo con Lipson (2000), si el "vivir con la enfermedad" es mucho más importante para los pacientes y por otro lado desligarse de las implicaciones sociopolíticas es negar una parte central de la enfermedad, la justificación de rescatar esos otros mundos de experiencia resultaría más que evidente. Años después los trabajos de antropólogos y otros científicos sociales sobre los asuntos de salud y enfermedad enfocados desde esta perspectiva crecían de manera exponencial, emergiendo dentro del seno de la Medicina este movimiento que aboga por rescatar la palabra y la narrativa del paciente. En la última década se ha acrecentado la atención y el recuento de las versiones de los pacientes (Morris, 200o) en lo que Polkinghorne (1988) denominaría como "giro narrativo" en el campo de la medicina y que alcanzaría ámbitos marcados por su carácter esencialmente clasificatorio como el DSM-IV, donde ya se señaló en su proyecto de reforma que se adolecía de una información imprescindible: la historia del paciente, su narrativa (Tucker, 1998). La medicina vuelve -o algunos pretenden que vuelva- a encontrar espacio para su alma humanística.

\section{Estudio de caso: los Trastornos Límite de Personalidad, de un enfoque convencional a un enfoque "narrativo"}

Concentraremos ahora nuestra atención en el caso de un trastorno, el Trastorno Límite de Personalidad o Borderline, y concretamente cómo ha sido conceptualizado, acotado y abordado desde la práctica clínica de la biomedicina y la psiquiatría más convencionales. También mostraremos el modo en que un grupo de investigadores apostaron por desarrollar un proyecto de investigación desde una mirada antropológica y con un enfoque narrativo. Nos referimos al proyecto "Del cuerpo a un mundo que tiembla. Narrativas, significados y experiencias en torno al Trastorno Límite de la Personalidad" realizado en comunidades de las regiones españolas de Extremadura y Castilla-La Mancha durante los años 2011 y 2012.

En primer lugar, perfilaremos sintéticamente las líneas y perfiles más evidentes con que estas versiones 
hegemónicas e institucionales han pensado y actuado con aquellas personas diagnosticadas con este trastorno. No nos guía una pretensión de exhaustividad, sino que resaltaremos algunos aspectos del mismo relacionados con nuestro argumento posterior.

Este Borderline (DSM-IV 301.831) o Trastorno Límite de Personalidad, es conocido también como trastorno límite o fronterizo. La definición del Trastorno Límite de la Personalidad parte del trabajo de Gunderson y Singer (1975) y se liga a la clasificación del Manual Diagnóstico y Estadístico de los Trastornos Mentales (APA, 2002). Se le define como "un trastorno de la personalidad que se caracteriza primariamente por inestabilidad emocional, pensamiento extremadamente polarizado y dicotómico y relaciones interpersonales caóticas”. Se incluye dentro del grupo B de trastornos de la personalidad, los llamados "dramático-emocionales". Es uno de los trastornos mentales que la literatura más ha suscitado, aunque destaca como muy poco de ella se encuentra sustentada en investigación empírica (Koenigsberg et al., 2002). La literatura contemporánea y diversos grupos de trabajo han puesto en cuestión la pertinencia de la denominación y la adscripción del diagnóstico. Afectando a alrededor de un $2 \%$ de la población (Torgersen et al., 200o) es el más frecuente de los trastornos de la personalidad (Gunderson; Zanarini, 1987). La característica principal de este trastorno es un esquema de comportamiento y funcionamiento alterado de forma estable y duradera, un patrón persistente de inestabilidad en las relaciones interpersonales, el afecto y la autoimagen y un escaso control de los impulsos. Se dice de sus afectados que poseen un sentido inestable de la personalidad y mecanismos de defensa inmaduros (Flores; Soto; Sánchez, 2007).

Su diagnóstico, manejo y tratamiento son uno de los retos más difíciles con los que se enfrenta la salud mental. La mayoría de pacientes cumplen criterios diagnósticos para otros trastornos de la personalidad (Zanarini et al., 1999). Diagnósticos paralelos como depresión mayor en el 71\% (Linehan, 1993), distimia en un $63 \%$ o trastorno de pánico en un 50\% (Bateman; Fonagy, 1999). De acuerdo con las cifras del DSM-IV-TR, tres de cada cuatro diag- nosticados son mujeres, (Torgersen et al., 2000; Zanarini et al., 1999). El TLP es un trastorno de inicio temprano y de curso crónico y existe consenso en la bibliografía sobre el elevado perfil de riesgo de estos pacientes con altas tasas de conductas suicidas. Los pacientes aquejados de TLP se muestran dependientes y hostiles, con escaso manejo de la frustración, que desemboca en episodios de ira y en ocasiones formas de violencia contra el self.

Se considera que el tratamiento más eficaz para estos pacientes es la combinación de abordajes psicológicos y farmacológicos, con la finalidad de aliviar los síntomas y las conductas que más alteran su calidad de vida. No obstante, ninguno de los dos tratamientos - ni por separado, ni combinados - ha demostrado suficiente eficacia terapéutica y a menudo han generado controversia.

El proyecto "Del cuerpo a un mundo que tiembla. Narrativas, significados y experiencias en torno al Trastorno Límite de la Personalidad" lo desarrollamos en dos hospitales, uno de Extremadura y otro de Castilla-La Mancha, durante los años 2011 y 2012. A las dos semanas de nuestra solicitud, recibimos los permisos para iniciarlo en el hospital extremeño. En el hospital castellano-manchego, el Comité Ético de Investigación nos negó el permiso. Esto retrasó nueve meses, hasta su final aprobación, tras una nueva solicitud enfatizando todavía más el carácter de investigación social y cualitativa que nos interesaba realizar. El núcleo del problema o malentendido estaba en que al pedir para trabajar con pacientes del hospital, sólo concebían la investigación como un ensayo clínico, y el proyecto que enviábamos estaba planteando trabajar con las narrativas de pacientes, con metodología etnográfica y cualitativa, pero sin ninguno de los requisitos mínimos de una investigación clínica experimental: se entendía que era una mala o inapropiada investigación al no ser clínica.

En el proyecto inicial, que fue rechazado, en el apartado de metodología explicábamos que:

La metodología de esta investigación es netamente cualitativa, ligada a presupuestos y las herramientas tradicionales del trabajo de campo etnográfico - Velasco y Díaz de Rada (2006). Para la producción de los observables empíricos - de acuerdo a los objetivos y el encuadre teórico donde esta investigación 
se inserta - se emplearán diversos tipos de relaciones de encuesta, incluyendo conversaciones informales, entrevistas semiestructuradas y entrevistas en profundidad. Las guías de observación y la elaboración de cuestionarios se perfilarán de acuerdo a la necesaria reflexividad refleja que tengan en cuenta tanto los objetivos de la investigación como el contexto de producción del conocimiento. La producción de narraciones sobre la experiencia de aflicción y el universo simbólico y de sentido desde la experiencia del enfermar requiere de relaciones de encuesta que se auxilien en el método biográfico. De igual forma, se trabajará en el análisis de discursos producidos en otros contextos u otras fuentes documentales, como cartas, diarios o cualquier tipo de anotación ligada a los intereses de esta investigación (Juárez et al., 2011).

A pesar de ello, en la carta que nos comunicaban la resolución de 9 de marzo de 2011 se decía:

Le comunico que este Comité Ético de Investigación Clínica, en la sesión celebrada el pasado 8 de marzo de 2011, ha entendido que dicho estudio no se ajusta a los criterios metodológicos estándar y por tanto, ha decidido su informe desfavorable.

La principal objeción que llegaron a verbalizarnos era que cómo íbamos a validar la información que nos proporcionarían los pacientes. Al no especificar en nuestro proyecto ninguno de los procedimientos estandarizados de la investigación cuantitativa y experimental clínica - como la encuesta tipo "multiple choice" o en el ensayo clínico -, automáticamente el Comité pensaba que carecíamos de la más mínima metodología o justificación científica.

Cuando dos años después regalamos un ejemplar de nuestro libro - con un primer avance de resultados del proyecto - a un colega psicólogo clínico, tras su lectura, días después manifestaba su sorpresa por qué el corazón de los "datos” fueran las historias de vida y narrativas de pacientes - cada uno de los tres capítulos nucleares están centrados en la historia de vida de una de estas personas. A continuación nos expresaba una inquietud-cuestionamiento relacionado con esta idea de "validación”: “¿Cómo estábamos prestando atención a la literalidad de unos discursos y narrativas producidas por sujetos "enfermos”? ¿Acaso conferíamos algún tipo de "verdad" a lo que esas voces enunciaban, unas voces que estaban generando una fantasía o elaborando un discurso "falso" fruto de su enfermedad?" Nuestra contestación fue que él estaba partiendo de la premisa de que esas narrativas las producían sujetos enfermos y tenían un carácter mítico o fantástico, que las invalidaban para un trabajo objetivo y científico con ellas. Nosotros partíamos de una premisa distinta: los enunciadores de esas narrativas eran personas que sufrían y que nos contaban con honestidad retazos o fragmentos de sus vidas cotidianas atravesadas por esa experiencia de aflicción, y por tanto considerábamos relevante registrar, analizar e interpretarlas - no nos investíamos del rol de inquisidores, censores o jueces, ni asumíamos las funciones de notarios que levantan acta de "realidad" que en ocasiones parecen asumirse desde algunos colegas de bata blanca. En otras palabras tomábamos tan en serio a esos "otros" como al discurso que los expertos elaboran - y elaboramos - sobre ellos. Quizás ésta sea una de las diferencias que separan la perspectiva clínica de la perspectiva de las ciencias sociales y humanas, y concretamente de una mirada antropológica o empapada por el "giro narrativo".

En esta investigación se muestra la tensión tradicional entre el empirismo y las aproximaciones socioconstructivistas. Pero, ¿cuáles son las aportaciones del giro narrativo y la NMB a la práctica clínica? ¿En qué ayuda esta perspectiva al proceso de ampliar la noción de evidencia en la clínica médica para dar cabida a los relatos de aflicción de pacientes y familiares? ¿Qué aporta la imperiosa necesidad de incluir entre los relatos "autorizados" y hegemónicos - "de bata blanca” - sobre el TLP, las voces de aquellos que sienten y padecen el trastorno? ¿De qué sirve ampliar el contexto de enfermedad de los neurotransmisores a la vida?

La perspectiva humanística aporta un conocimiento en forma de evidencia que se traduce en una mejora de la atención y cuidados. Así, por ejemplo, estas narrativas arrojan un sentido del padecimiento construido en torno al sufrimiento y la incomprensión. "Saber" se convierte en un asunto secundario cuando se adentran en el "comprender": las narrativas de los entrevistados supuraban sufrimiento, asunto central en la elaboración de la trama. Parte de ese sufrimiento derivaba de las 
manifestaciones clínicas suficientemente descritas en la bibliografía. Las tramas se abren a episodios complejos de conflictos con familiares o compañeros de trabajo, búsquedas incansables sobre explicaciones o curas en forma de "pociones mágicas". Pero en todos estos relatos, el asunto central es la incomprensión. El desdén de profesionales sanitarios durante los internamientos, la incomprensión de familiares y amigos. Otorgar un lugar protagónico a estos relatos permite dirigir mejor las intervenciones de los profesionales. A modo de ejemplo, la mayor parte de las narrativas de los entrevistados habían encarnado uno de los reclamos de la terapia recibida en las consultas de salud mental: lo importante era la vida ordenada, entendida aquí como la clave de la recuperación, unos horarios y actividades marcadas por la disciplina. Desde nuestro punto de vista, más allá de la crítica sobre la emanación de control social y moral que transmiten estos postulados, estas demandas se convertían en una fuente de estrés. Pero poco se hacía para trabajar el sentimiento de "incomprensión". El reclamo por la vida ordenada parte de una concepción estándar del padecimiento y su terapia -una perspectiva casi de magia simbólica y moralista que asume que el orden interior es un reflejo del orden en la vida cotidiana-y las preocupaciones obvias de los que lo sufren: las demandas por que los que les rodean les entiendan.

$\mathrm{Al}$ entender los relatos de aflicción como evidencias para la clínica, aquellos se tornan en documentos auditores de la calidad asistencial, permitiendo la evaluación y la mejora de las prácticas al incorporar la "comprensión” del trastorno. Así, por ejemplo, hemos podido rescatar alguna de las contradicciones de la atención sanitaria durante los internamientos en las unidades de salud mental. Generalmente estas unidades se caracterizan por el férreo control de los pacientes, un "régimen carcelario" en palabras de algunas de las entrevistadas. Los protocolos de seguridad son entendidos como impedimentos para conseguir una estancia autónoma sin perder la identidad, aunque la mayor parte de los relatos los consideraba ineficaces: siempre había una forma de saltarlos. Lo que pudiera contribuir a la seguridad de los pacientes se enfrenta luego con las prácticas y comentarios de los profesionales, que se dirigen a ellos o comentan sobre ellos siempre bajo la idea de que lo único que persiguen en "llamar la atención". Nada más alejado de aquel "detached concern" al que antes nos referíamos y que rendiría en términos de calidad asistencial. De nuevo, comprender la enfermedad -la trama de "falta de comprensión-atención" - más allá de saber de la misma. En el ámbito del riesgo y la seguridad, el sentido del padecimiento puede arrojar una luz diferente sobre la perspectiva del saber. Así, los significados otorgados a las prácticas autolesivas, por ejemplo, difieren de la idea de "riesgo" del personal médico. En nuestras entrevistas, algunos comentaban un inicio en ellas por mera imitación de lo que habían visto curiosamente en algún internamiento anterior. Pero otros relatos explicaban el carácter "terapéutico" de las mismas. Ante una sensación de vacío infinito, el dolor las devolvía al mundo. Al individualizar los sentidos - y no tanto las prácticas - la forma individualizada de abordaje debería tener un mejor desarrollo. María nos confesaba que en muchas ocasiones esas prácticas estaban motivadas por la actitud "carcelaria" e hipervigilante del personal sanitario, una forma de respuesta a lo que ella consideraba una agresión a su libertad o intimidad, incluso al robo de su identidad durante los ingresos. Por supuesto, las prácticas de vigilancia y control parecen necesarias, pero esta atención a los sentidos individuales y el diálogo parecen rendir mucho más que el enfrentamiento con los férreos y estandarizados protocolos. Al tratar la vida y no el diagnóstico, todo se vuelve más complejo. Estas narrativas son, por así decirlo, el reverso de los historiales médicos que con tanto celo guardan los hospitales y los profesionales médicos. La historia de vida dentro de la historia clínica; huellas del impacto de la enfermedad y el diagnóstico en la vida. En nuestra opinión, la información contenida en los historiales médicos - las evidencias tradicionales - aceptan de manera tácita la amputación de esos otros órdenes de significados que se vuelven centrales en las narrativas de aflicción. Y desde el momento que se acepta la importancia en las tramas de los aquejados, debe aceptarse su lugar protagónico en la práctica médica. 
El asunto se reduce entonces a cuestiones de calidad asistencial (Juárez; Martos; Cipriano, 2013, p. 99-103). La búsqueda de este sentido no sólo resulta interesante para antropólogos médicos u otros científicos sociales interesados en este campo. Muy al contrario - al menos desde la óptica de la Medicina Basada en Narrativas (Juárez; Cipriano, 2012) -, constituye una evidencia de primer orden para la práctica clínica y asistencial, también en la salud mental (Launer, 1999). Tal y como señala Hydén (1997, p. 49), "las narrativas de los pacientes dan voz al sufrimiento en una forma que queda fuera del dominio de la voz de la biomedicina”, pero quizás haya llegado el momento de que esos dominios se ensanchen para ofrecer ese espacio.

\section{A modo de conclusión}

Desde la perspectiva de las Ciencias Sociales y las Humanidades, son percibidos nuevos problemas y abordajes de fenómenos, procesos y relaciones sociales (Ianni; Barreto Jr; Martins, 2013, p. 10), y por ello resultan estratégicas en el campo sanitario y de la salud pública. Nos ayudan a identificar campos y temas emergentes que reclaman nuestra atención y ponen el foco en actores protagonistas, habitualmente ninguneados o silenciados: en este caso los "enfermos".

Frente a los discursos que pretenden enterrar las aportaciones de las $\mathrm{SHH}$, defendemos la revisión de las nociones de "impacto", "evidencia” o "trasferencia de resultados" para valorar aquellas en su justa medida. Más allá de las miopes y cortoplacistas lógicas del mercado, las aportaciones de las ciencias humanas mantienen hoy un protagonismo central. Hemos revisado aquí los impactos y reelaboraciones que la Medicina ha recibido en el último medio siglo, traducidas en mejoras de asistencia y/o reducciones del gasto que pocas veces son puestas sobre la mesa de los balances. El giro narrativo y la vuelta a la esencia humanista en el otrora templo de la razón empírica es un argumento para construir nuevas definiciones y criterios para aquellas categorías.

No obstante, esta mudanza paradigmática de los últimos años, donde la estadística empieza a ser acompañada en un nuevo empirismo con investigaciones que privilegian los relatos de aflicción y otorgan un incipiente protagonismo de los sujetos, sigue siendo minoritaria y tiene un desigual impacto en según qué países. Resulta paradójico que sea en los países que encarnan el mainstream de los modelos más convencionales de la medicina - los países anglosajones y especialmente EE.UU. - que apuestan por la Medicina Basada en la Evidencia y con altas inversiones en tecnología, donde la penetración e influencia de esta mudanza epistemológica perfilada en las páginas anteriores ha sido mayor. Probablemente porqué fue allí precisamente donde surgió dentro de la profesión médica la Medicina Basada en Narrativas como reacción a la tiranía y corto alcance de la Medicina Basada en la Evidencia.

Somos conscientes de que en los países de la Europa del Sur como España, y también en los países iberoamericanos, queda todavía una larga senda por recorrer en aumentar el impacto epistemológico, teórico, y en intervención y políticas públicas en una medicina menos abierta a la escucha y la observación desde una perspectiva social y humanista: la influencia - e incluso la visibilidad - de este paradigma alternativo y giro epistemológico ha sido mucho menor.

De todos modos, inclusive en los países y contextos más receptivos a estas nuevas propuestas y realidades, no debemos ilusionarnos en exceso con el alcance de este cambio epistemológico experimentado en algunos segmentos de la medicina como técnica científica. En la actualidad, son pocos y desde luego no ocupan posiciones hegemónicas ni influyentes los que se vinculan al pensamiento crítico e incorporan los riesgos de estar cautivos de la falacia del alcance ilimitado de la tecnología para llevar el bienestar y la salud a la población. Por el contrario, amplios sectores de la profesión y del mercado médico han hecho caso omiso a estas nuevas perspectivas, y siguen equiparando acceso a la salud y consumo de tecnología, fomentando y justificando la inversión - casi exclusiva - en tecnologías. Unas tecnologías que a día de hoy no están calibradas ni dirigidas a otorgar sentido a los padecimientos, ni a aliviar el sufrimiento de las personas.

\section{Referencias}

ACKERKNECHT, E. La médicine hospitaliére a

Paris (1794-1848). Paris: Payot, 1986. 
APA - AMERICAN PSYCHIATRIC ASSOCIATION. DSM-IV-TR. Manual diagnóstico y estadístico de los trastornos mentales. Barcelona: Masson, 2002.

ARONSON, J. K. Autopathology: the patient's tale. $B M J$, London, v. 321, n. 7276, p. 1599-16o2, 2000.

BATEMAN, A.; FONAGY, P. Effectiveness of partial hospitalisation in the treatment of borderline personality disorder: a randomised controlled trial. American Journal of Psychiatry, Arlington, v. 156, n. 10, p. 1563-1569, 1999.

BERGER, P.; LUCKMANN, T. La construcción social de la realidad. Buenos Aires: Amorrortu, 1986.

BOURDIEU, P. Comprender. In: BOURDIEU, P. La miseria del mundo. Madrid: Akal, 1999. p. 527-557.

BRUNER, J. Actual minds, posible worlds. Cambridge: Harvard University Press, 1986.

BURY, M. Health and illness in a changing society. London: Routledge, 1997.

BURY, M. Illness narrative: fact or fiction? Sociology of Health \& Illness, Malden, v. 23, n. 3, p. 263-285, 2001.

CANGUILHEM, G. Idéologíe et rationalité dans l'histoire des sciences de la vie. Paris: Librairie Philosophique J. Vrin, 1993.

CARRIÓ, S. Medicina narrativa: una estrategia para formar profesionales reflexivos. Conexión Pediátrica, Buenos Aires, v. 1, n. 3, p., 2008. Disponible en: <http://www.conexionpediatrica.org/index.php/ conexion/article/view/91/103>. Acceso en: 9 oct. 2015.

CHARON, R. Medical interpretation: implications of literary theory of narrative for clinical world. Journal of Narrative Life History, Massachusetts, v. 3, n. 1, p. 79-9, 1993 a.

CHARON, R. The narrative road to empathy. In: SPIRO, H. et al. (Ed.). Empathy and the practice of medicine: beyond pills and the scalpel. New Haven: Yale University Press, 1993b. p. 147-159.

CHARON, R. Narrative medicine. A model for empathy, reflection, profesión and trust. Journal of the American Medical Association, Chicago, v. 286, n. 15, p. 1897-1902, 2001.

CHARON, R. From detached concern to empathy: humanizing medical practice (review). Journal of
Health Politics, Policy and Law, Durham, v. 28, n. 6, p. 1121-1125, 2003.

CHARON, R. Narrative medicine: honoring the stories of illness. New York: Oxford University Press, 2006.

CHARON, R. et al. Narrative evidence based medicine. The Lancet, London, v. 371, n. 9609, p. 296-297, 2008.

COMAROFF, J.; COMAROFF, J. L. The madman and the migrant: work and labor in the historical consciousness of a South African people. American Ethnologist, New York, v. 14, n. 2, p. 191-209, 1987.

COMELLES, J. M. El regreso de las culturas: diversidad y práctica médica en el siglo XXI. In: JUÁREZ, G. F. (Coord.). Salud e interculturalidad en América Latina: perspectivas antropológicas. Quito: Abya Yala, 2004. p. 17-30.

ENTRALGO, P. Historia de la medicina. Barcelona: Masson, 1978.

EVANS, M.; SWEENEY, K. The human side of medicine: occasional paper OP76. London: Royal College of General Practitioners, 1998.

EVENS, M. Two kinds of rationality. Minneapolis: University of Minnesota, 1995.

FLORES, T.; SOTO, Á.; SÁNCHEZ, C. Trastorno límite de la personalidad: a la búsqueda del equilibrio emocional. Barcelona: Morales i Torres, 2007.

FOUCAULT, M. El nacimiento de la clínica: una arqueología de la mirada médica. Madrid: Siglo XXI, 2007.

GEERTZ, C. El antropólogo como autor. Barcelona: Paidós, 1989.

GEERTZ, C. Géneros confusos: la refiguración del pensamiento social. In: GEERTZ, C. et al. $\varepsilon l$ surgimiento de la Antropología Posmoderna. Barcelona: Gedisa, 1991. p. 63-77.

GEERTZ, C. La interpretación de las culturas. Barcelona: Gedisa, 1992.

GEERTZ, C. Conocimiento local: ensayos sobre la interpretación de las culturas. Barcelona: Paidós, 1994. 
GOOD, B. J. The heart of what's the matter: the semantic of illness in Iran. Transcultural Psychiatric Review, Montreal, v. 19, n. 1, p. 75-92, 1977.

GREENHALGH, T. Why study narrative? BMJ, London, v. 318, n. 7175, p. 48-50, 1999.

GREENHALGH, T.; HURWITZ, B. Narrative based medicine in an evidence based world: dialogue and discourse in clinical practice. London: BMJ Books, 1998.

GUNDERSON, J.; SINGER, M. Defining borderline patients: an overview. American Journal of Psychiatry, Arlington, v. 132, n. 1, p. 1-10, 1975.

GUNDERSON, J.; ZANARINI, M. Current overview of the borderline diagnosis. Journal of Clin Psychiatry, Memphis, v. 48 (Suppl), p. 5-11, ago. 1987.

GUYATT, G. Preface. In: GUYATT, G.; RENNIE, D. (Ed.). User's guide to the medical literature: essentials of evidenced medicine clinical practice. [S.l.]: AMA Press, 2002. p. 13-16.

HALPERN, J. From detached concern to empathy: humanizing medical practice. New York: Oxford University Press, 2001.

HERNÁEZ, Á. Antropología médica: teorías sobre la cultura, el poder y la enfermedad. Barcelona: Anthropos Editorial, 2008.

HUNTER, K. Doctor's stories: the narrative structure of medical knowledge. Princeton: Princeton University Press, 1991.

HURWITZ, B. Narrative and the practice of medicine. The Lancet, London, v. 356, n., p. 20862089, 2000.

HYDÉN, L-C. Illness and narrative. Sociology of the health and the illness, Malden, v. 19, n. 1, p. 48-69, 1997.

IANNI, A. M. Z.; BARRETO JR, I. F.; MARTINS, C. L. A produção do conhecimento na interface entre as Ciências Sociais e a Saúde Pública/Coletiva. Saúde e Sociedade, São Paulo, v. 22, n. 1, p. 9-11, 2013.

ILLICH, I. Némesis médica. México, DF: Editorial Joaquín Mortíz, 1978.

JUÁREZ, L. M. Palabras, significados y políticas de representación en la arena de la interculturalidad en salud. Los espacios de la cultura en la atención al hambre y la desnutrición en el oriente de Guatemala. Nuevo Mundo Mundos Nuevos, París, v. , n. , p. , 2011. Disponible en: <http:// nuevomundo.revues.org/61563>. Acceso en: 13 oct. 2015 .

JUÁREZ, L. M. et al. Del cuerpo a un mundo que tiembla: narrativas, significados y experiencias en torno al Trastorno Límite de la Personalidad. Proyecto de Investigación, 2011.

JUÁREZ, L. M.; CIPRIANO, C. Medicina y narrativas: de la teoría a la práctica. Almería: Editorial Círculo Rojo, 2012.

JUÁREZ, L. M.; MARTOS, J. A. F.; CIPRIANO, C. Nosotros, los trastornos límite de personalidad: narrativas y relatos de aflicción. Almería: Editorial Círculo Rojo, 2013.

JUÁREZ, L. M.; RODRÍGUEZ, B.; CONDE, D. Cuidados basados en narrativas: redefiniendo la jerarquía de la evidencia. Index de Enfermería, Granada, v. 22, n. 1-2, p. 55-59, 2013. Disponible en <http://www.index-f.com/index-enfermeria/v22n12/7977.php>. Acceso en: 13 oct. 2015.

KALITZKUS, V. Narrative-based medicine: potential, pitfalls, and practice. The Permanent Journal, Portland, v. 13, n. 1, p. 8o-86, 2009.

KLEINMAN, A. Medicine's symbolic reality: on the central problem of philosophy of medicine. Inquiry, Colchester, v. 16, n. 1-4, p. 55-65, 1973.

KLEINMAN, A. Patients and healers in the context of culture: an exploration of the borderlands between Anthropology, Medicine and Psychiatry. Berkeley: University of California Press, 1980.

KLEINMAN, A. The illness narratives: suffering, healing and the human condition. London: Basic Books, 1988.

KOENIGSBERG, H. et al. Characterizing affective instability in borderline personality disorder. The American Journal of Psychiatry, Arlington, v. 159, n. 5, p. 784-788, 2002.

LAUNER, J. Narrative based medicine: a narrative approach to mental health in general practice. 
$B M J$, London, v. 318, n. 7176, p. 117-119, 1999.

LINEHAN, M. Cognitive-behavioral treatment of borderline disorder. New York: The Guildor Press, 1993 .

LIPSON, J. Cultura y cuidados de enfermería. In: XIV COLOQUIO NACIONAL DE INVESTIGACIÓN EN ENFERMERÍA, 14., 1999, Tunja. Conferencia... Tunja: Index de Enfermería, 200o, p. 28-29. Disponible en: <http://www.index-f.com/indexenfermeria/28-29revista/28-29_articulo_19-25. php>. Acceso en: 13 oct. 2015.

MALINOWSKY, B. Practical anthropology. Journal of the International African Institute, Cambridge, v. 2, n. 1, p. 22-38, 1929. Disponible en: <http:// www.jstor.org/stable/1155162>. Acceso en: 13 oct. 2015 .

MENÉNDEZ, E. Hacia una práctica médica alternativa: hegemonía y autoatención (gestión) en salud. México: Casa Chata-Ciesas, 1983.

MENÉNDEZ, E. La enfermedad y la curación. ¿Qué es medicina tradicional? Alteridades, México DF, v. 4, n. 7, p. 71-83, 1994.

MORRIS, D. How to speak postmodern: Medicine, illness, and cultural change. The Hastings Center Report, Garrison, v. 3o, n. 6, p. 7-16, 2000.

PERDIGUERO, E.; COMELLES, J. M. (Ed.).

Medicina y cultura: estudios entre la antropología y la medicina. Barcelona: Bellaterra, 2000.

POLKINGHORNE, D. Narrative knowing and the human sciences. Albany: State University of New York Press, 1988.

PORTER, R. The greatest benefit to mankind: a medical history of humanity from antiquity to the present. London: Harper Collins, 1997.
ROSEN, G. De la policía médica a la medicina social. México: Siglo XXI, 1985.

SACKETT, D. A primer on the precision and accuracy of the clinical examination. JAMA, Chicago, v. 267, n. 19, p. 2638-44, 1992.

SACKETT, D. Evidence based medicine: how to practice and teach evidence based medicine. London: Wolfe Publishing LTD, 2009.

SACKETT, D. et al. Evidence based medicine: what it is and what it isn't. BMJ, London, v. 312, n., p. 71-72, 1996.

SACKETT, D. et al. Choosing the best research design for each question. BMJ, London, v. 315, n., p. 1636-40, 1997.

SILVA, S. et al. The marriage of evidence and narrative: scientific nurturance within clinical practice. Journal of Evidence in Clinical Practice, Malden, v. 17, n 4., p. 1-9, 2010.

TORGERSEN, S. et al. A twin study of personality disorders. Comprehensive Psychiatry, Philadelphia, v. 41, n. 6, p. 416-425, 2000.

TUCKER, G. Putting DMS-IV in perspective. The American Journal of Psychiatric, Arlington, v. 155, n. 2, p. 159-161, 1998.

TURNER, V.; BRUNER, E. The Anthropology of experience. Chicago: University of Illinois Press, 1986.

ZANARINI, M. et al. Violence in the lives of adult borderline patients. The Journal of Nervous and Mental Disease, Philadelphia, v. 187, n. 2, p. 65-71, 1999.

\section{Contribución de los autores}

Los dos autores han trabajado de manera similar en todas las fases de la investigación, desde la revisión de bibliografía, el diseño y la delimitación de objetivos, el trabajo de campo y la redacción del artículo.

\section{Agradecimientos}

Los autores agradecen las sugerencias de los evaluadores anónimos de Saúde e Sociedade, que han ayudado a mejorar el presente artículo.

Recibido: 15/01/2015

Presentado: 03/09/2015

Aprobado: 22/09/2015 